\title{
Enhancing evaporative mass transfer and steam stripping using microwave heating
}

O. Ogunniran, E.R. Binner, A.H. Sklavounos and J.P. Robinson*

Faculty of Engineering, University of Nottingham. NG7 2RD. UK

\begin{abstract}
The effect of microwave heating on evaporative mass transfer of hydrocarbons was investigated for a number of contaminated solid materials. The rate of oil removal could be rationalised by the velocity of steam that was created by selective heating of water within the solid. A single correlation was found to fit 45 independent experiments across 10 separate variables, and the correlation was consistent with the physics of evaporative mass transfer. It is shown for the first time that steam stripping is the dominant mechanism that governs hydrocarbon removal during microwave processing. It was further discovered that mass transfer is enhanced due to microwave heating when compared to conventional stripping processes, with this improvement in efficiency due to the ability of the microwave process to overcome the channelling effects that limit conventional mass transfer processes.
\end{abstract}

\section{Introduction}

Hydrocarbon-containing soils pose a threat to health and to the environment. They arise from legacy industrial activity, where hydrocarbon containing wastes were disposed of with minimal treatment. These are typically found on industrial complexes, fuel storage and coal-gas sites and also within harbour sediments. In these cases there is a need to remediate the legacy hydrocarbon contamination via in-situ or ex-situ treatment processes. More recent industrial processes that produce hydrocarbon contaminated solids occur within the oil \& gas and steel industries, with modern practice and legislation requiring that these wastes are effectively treated to remove the hydrocarbon phase prior to their disposal or reuse. In most cases, whether for legacy sites or current industrial practice, the technologies used to separate the contaminant hydrocarbons are based on thermal desorption or gas stripping. Electrical heating and air stripping have been explored for in situ remediation of contaminated soil [1-2], and thermal desorption of oil-contaminated drill cuttings via a heated screw conveyor is widely practised within the oil industry [3]. Raising the temperature of the solid increases desorption [4] due to an increase in the vapour pressure of the hydrocarbons relative to the partial pressure within the contacting gas. 
Microwave heating has been studied as an alternative desorption technique for a number of applications, and it has been shown that this approach can be more energy efficient than conventional systems due to the mechanism through which microwave energy desorbs the hydrocarbon phase. The hydrocarbon and water phases coexist on the surface and within the pore structure of the solid [5]. During microwave processing it is only the water that absorbs microwaves, with steam generated insitu from within the capillaries and pores of the solid material. Previous studies have highlighted gains in efficiency with this approach for applications including contaminated drill cuttings, contaminated soils and oil sands. A number of studies have speculated that entrainment, steam stripping or steam distillation are possible mechanisms that explain empirical hydrocarbon removal results [6-11], but none have offered conclusive evidence to date. There is also ambiguity within the literature, with studies using terminology around steam stripping and steam distillation interchangeably, not recognising that they are based upon different physical principles. Steam distillation occurs when the boiling point of the hydrocarbon phase is reduced due to the presence of an immiscible phase, in this case water. With steam distillation the hydrocarbon is boiled, whereas with steam stripping the hydrocarbon is removed by evaporation into the stripping gas media. The aim of this study is to establish the extent to which steam-stripping occurs during microwave remediation of hydrocarboncontaminated solids by measuring mass transfer coefficients for hydrocarbon removal and comparing with existing mass transfer correlations.

Previous workers have studied the rate of mass transfer of hydrocarbons from soils to a gas stripping medium and derived empirical models relating mass transfer coefficient with the velocity of the stripping medium [12-14]. They studied the effect of introducing gas from an external source into a partially saturated contaminated soil media. Wilkins et al [12] noticed that the residual water content and water distribution affected the mechanism of hydrocarbon mass transfer into the stripping gas, which could either be governed by diffusive or convective transport. In this scenario, hydrocarbon droplets may become trapped between aqueous phases in small pores, restricting movement into the gas phase. This phenomenon is known as channelling, where the stripping medium bypasses the trapped organic phase, and is a common disadvantage of gas stripping in conventional fixed bed systems. Yoon et al [13] corroborated the findings of Wilkins et al [12] by showing that hydrocarbon mass transfer can become diffusion rate-limited, even at high gas velocities. Vander Ham and Brouwers [14] studied non-equilibrium mass transfer into a steam stripping medium, and found that the interfacial area between steam and the hydrocarbon phase may decrease during treatment of the contaminated soil. In all cases, hydrocarbon removal was confirmed to take place via an evaporative mass transfer process, with the bulk gas temperature being below the end-point of the hydrocarbon phase. 
Mass transfer coefficients in fixed beds can be linked to the velocity of the stripping gas in a generalised correlation [15]:

$\mathrm{Sh}=\mathrm{bRe}^{\mathrm{c}} \mathrm{Sc}^{0.33}$

Where Sh is the Sherwood number, Re is the Reynolds number, Sc is the Schmidt number, b and c are constants determined empirically. The Sherwood and Reynolds Numbers are defined as:

$\operatorname{Sh}=\frac{k_{g} d_{p}}{D_{a}}$

$\operatorname{Re}=\frac{\rho \mathrm{ud}_{p}}{\mu}$

$\mathrm{k}_{\mathrm{g}}$ is the gas phase mass transfer coefficient, $\mathrm{d}_{\mathrm{p}}$ is the characteristic length (usually defined as the mean particle diameter), $D_{a}$ is the diffusion coefficient of the contaminant in the stripping medium, $\rho$ is the density of the stripping medium, $u$ is the velocity of the stripping medium and $\mu$ is the viscosity of the stripping medium.

The mass transfer coefficient can be estimated from Fick's law [16]:

$\mathrm{J}=\mathrm{k}_{\mathrm{g}}\left(\mathrm{c}_{\mathrm{i}}-\mathrm{c}_{\mathrm{b}}\right)$

where $\mathrm{J}$ is the net molar flux of the species desorbed from the fixed bed into the gas phase, $\mathrm{c}_{\mathrm{b}}$ is the concentration of the desorbed species in the bulk gas phase, and $\mathrm{c}_{\mathrm{i}}$ is the concentration of the desorbed species at the interface with the stripping gas, which is related to its vapour pressure. The partial pressure at the interface is assumed to equal the vapour pressure at the stripping temperature. Partial pressure values are subsequently converted to gas phase concentration $\left(\mathrm{mol} / \mathrm{m}^{3}\right)$ to yield $\mathrm{k}_{\mathrm{g}}$ values $(\mathrm{m} / \mathrm{s})$ when the molar flux is known or measured. Within fixed beds it is often not possible to measure molar flux due to uncertainties in the interfacial area of the solid particles within the bed, which typically exhibit a large size distribution and poorly-defined interstitial regions. In previous studies [12-14], the relationship between the mass transfer coefficient and gas velocity in fixed beds of soil has been presented in a modified version of Equation 1 as follows;

$\mathrm{Sh}_{0}=\mathrm{bPe}^{\mathrm{c}}$

where $\mathrm{Sh}_{\mathrm{o}}$ is the modified Sherwood number and Pe is the Peclet number 
The modified Sherwood number is defined as;

$\mathrm{Sh}_{0}=\frac{\mathrm{k}_{\mathrm{g}} \mathrm{d}_{\mathrm{p}}}{\mathrm{D}_{\mathrm{a}}}$

where $\mathrm{a}$ is the specific interfacial area $\left(\mathrm{m}^{2} / \mathrm{m}^{3}\right)$, and $\mathrm{k}_{\mathrm{g}}$ a is termed the overall mass transfer coefficient $\left(\mathrm{s}^{-1}\right)$, which is used when the interfacial area cannot be measured or estimated. Using this approach Equation 4 can be rewritten as:

$\dot{N}=k_{g} a V\left(c_{i}-c_{b}\right)$

Where $\dot{N}$ is the molar flowrate and $V$ the bed volume.

Peclet number $(\mathrm{Pe})$ is the ratio of the effectiveness of mass transport by bulk flow to mass transport by diffusion [17]:

$P e=f(R e, S c)=\frac{u d_{p}}{D_{a}}$

The Peclet number gives an indication of the type of flow in a low permeability environment, values of Pe less than 1 typically suggests creeping flow and a mass transfer rate mainly driven by diffusion. Values of 2 and above suggest that mass transfer is mainly governed by convective bulk transport.

\section{Hypothesis and Objectives}

It is proposed that hydrocarbon removal from contaminated solids under microwave heating occur due to steam stripping, with the steam generated in-situ during microwave heating. In this case the efficiency of oil removal will therefore be related to the velocity of the steam within the pores of the solid material, consistent with evaporative mass transfer processes governed by Equation 5. Steam distillation, the other candidate mechanism identified in previous studies, has no velocity-dependence as the oil \& water will be removed simultaneously by boiling. The objective of this work is to measure the hydrocarbon mass transfer coefficient and correlate this with steam velocity across a range of variables including power, heating frequency, oil content, water content, feedstock source and bed geometry. A positive correlation would strongly suggest that steam stripping is the dominant mechanism. If hydrocarbon removal is independent of velocity then this would imply that the steam distillation mechanism is dominant. 


\section{Experimental}

\section{Feedstock}

The samples used for this study were oil-contaminated drill cuttings obtained from drilling operations within the southern sector of the North Sea. Three different samples were studied, two Shale-based and one Sandstone-based. Their properties are detailed in Table 1.

\section{Microwave heating setup}

Two separate microwave heating systems were employed in order to yield steam velocities from 0.1$10 \mathrm{~m} / \mathrm{s}$, thereby spanning three orders of magnitude. System A, shown in Figure 1 comprised a $110 \mathrm{~mm}$ diameter TM cavity and applied power levels of $1-5 \mathrm{~kW}$ at $2.45 \mathrm{GHz}$. A 3 -stub tuner and a piston tuner were used to reduce reflected power by matching the impedance between the cavity/feedstock and the generator. $70 \mathrm{~g}$ of sample was placed within a $76 \mathrm{~mm}$ diameter quartz reactor. Nitrogen was passed through the cavity at 5 litres/min to provide an inert atmosphere. Extracted vapours were then passed through a condenser and a scrubber before venting to atmosphere through an extraction unit. Steam velocities of $0.1-0.9 \mathrm{~m} / \mathrm{s}$ were attainable with this processing system.

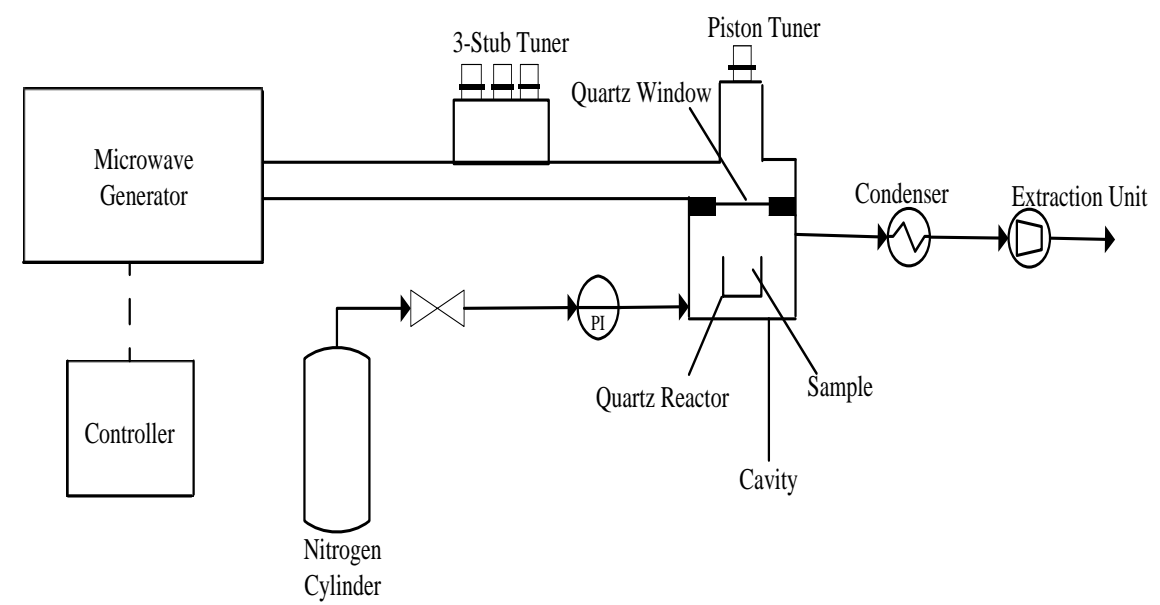

Figure 1: Schematic of the single mode microwave treatment experimental set-up (System A)

A second microwave system (System B) was used to yield steam velocities ranging from 6-10 m/s, shown in Figure 2. 


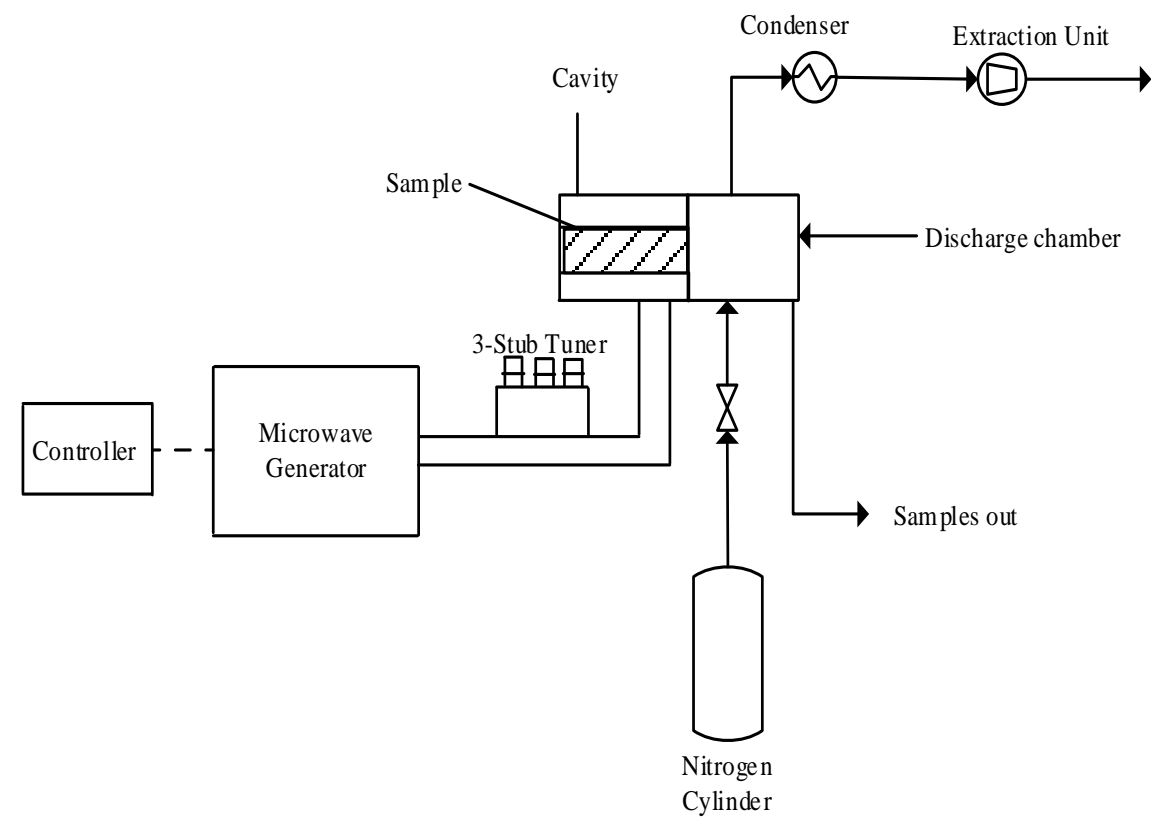

Figure 2: Schematic of a fixed bed pilot scale microwave system (System B)

$6.5 \mathrm{~kg}$ samples were charged within a horizontal cylindrical bore within a ceramic block of $400 \mathrm{~mm}$ length and $100 \mathrm{~mm}$ diameter. A $100 \mathrm{~kW}$ microwave generator operating at $896 \mathrm{MHz}$ was used to deliver power to the cavity, with a 3 -stub tuner used to improve impedance matching. Steam and hydrocarbon vapours were removed from an adjacent chamber, through which the treated sample could also be removed. Nitrogen was passed through the discharge chamber at 5 litres/min to provide an inert atmosphere.

\section{Oil content and water content analysis}

The water content of each sample was determined using the Dean \& Stark method as documented in ASTM-D95. The residual water content before and after treatment was measured to determine the mass loss of water. Oil content was measured before and after microwave treatment by Soxhlet extraction with dichloromethane (DCM), and subsequent evaporation of the DCM.

\section{Porosity and bulk density}

The porosity of each sample was determined by measuring the bulk density and comparing the values with the skeletal density. The skeletal density was determined using an Accupyc 1330 gas pycnometer. Bulk density was calculated from the mass of sample within a $500 \mathrm{ml}$ volume. The initial oil and water content and the bulk density and porosity of the samples used in this study are given in Table 1. 
Table 1. Bulk density, porosity, and oil and water contents of the oil contaminated drill cuttings samples used in this study

\begin{tabular}{|l|l|l|l|l|}
\hline Sample & $\begin{array}{l}\text { Water content } \\
(\% \mathbf{w} / \mathbf{w})\end{array}$ & $\begin{array}{l}\text { Oil content } \\
(\boldsymbol{\%} \mathbf{w} / \mathbf{w})\end{array}$ & $\begin{array}{l}\text { Bulk density } \\
\left(\mathbf{k g} / \mathbf{m}^{\mathbf{3}}\right)\end{array}$ & Porosity \\
\hline Shale 1 & $9.5-14.0$ & $8.5-12.0$ & $1400-2100$ & $0.29-0.49$ \\
\hline Shale 2 & $7.0-8.0$ & $9.0-10.0$ & $1500-1600$ & $0.44-0.48$ \\
\hline Sandstone & $8.5-9.5$ & $7.5-8.5$ & $1900-2000$ & $0.30-0.34$ \\
\hline
\end{tabular}

Calculation of steam velocity and mass transfer coefficient

The residual oil and water content within the sample were measured for each experiment. Four values of heating time were investigated for each variable studied, and these values used to establish the rate of water and oil removal. Steam velocity was varied indirectly by changing the microwave power and the bed geometry. These were the primary control variables used within this study. Other variables included the feedstock porosity, oil and water content, which were measured but not controlled. Steam velocity through the sample bed was calculated based on the water removal rate, bed porosity and bed diameter:

$u=\frac{\Delta W}{A \varepsilon \rho \Delta t}$

Where $\Delta W$ is the change in water mass within the solid over time interval $\Delta t, \mathrm{~A}$ is the cross-sectional area of the heating cavity, $\varepsilon$ is the porosity of the bed and $\rho$ the density of steam, taken as the saturation value at $1 \mathrm{~atm}\left(0.59 \mathrm{~kg} / \mathrm{m}^{3}\right)$.. The overall mass transfer coefficient for oil, $k_{g} a$, was determined using Equation 7 using empirically-determined oil removal rates for different bed volumes. $c_{i}$ was estimated based on the vapour pressure of decene at $100^{\circ} \mathrm{C}(0.13 \mathrm{~atm})$ and $\mathrm{c}_{b}$, the hydrocarbon concentration within the bulk gas phase was assumed to be zero [18]. Decene was chosen as this is representative of the hydrocarbon phase used within the drilling fluid that is present within each feedstock. Whilst temperature was not measured, $100^{\circ} \mathrm{C}$ was used for steam density and vapour pressure calculations as this is consistent with the underpinning hypothesis of this study.

\section{Results and Discussion}

Forty five separate sets of experiments were carried out, with each set consisting of at least four heating times. The oil and water remaining within the soil was measured after each heating test, with example data shown in Figure 3. 

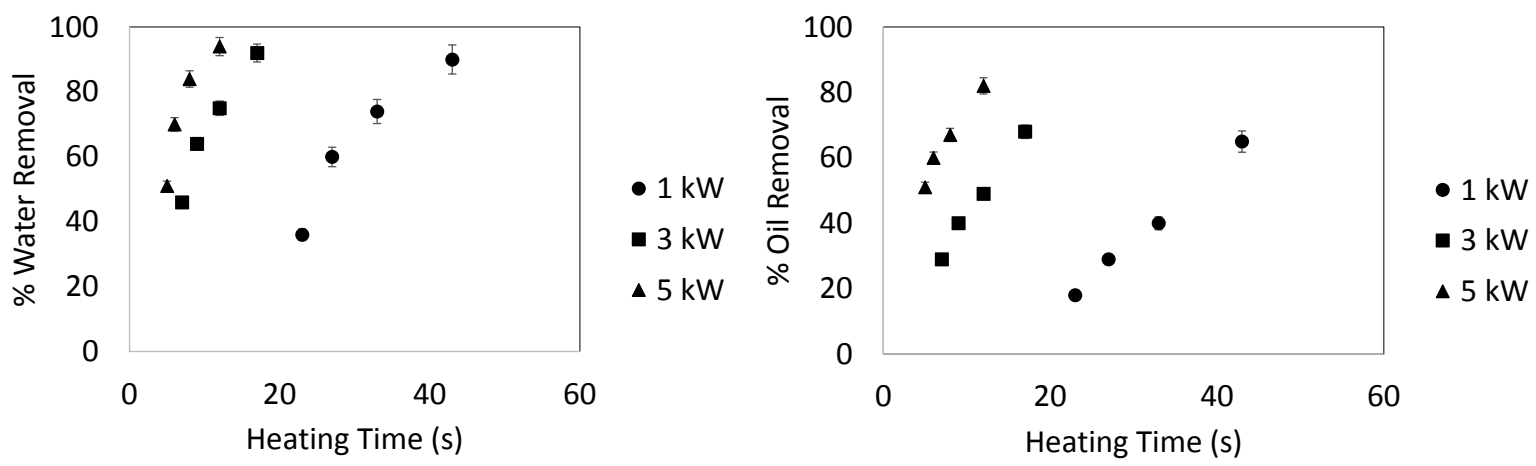

Figure 3 - Water and Oil removal plotted against time at power levels of 1-5kW. Data obtained with System A using Shale 1; $12 \%$ water, $9 \%$ oil,bulk density $=1650 \mathrm{~kg} / \mathrm{m}^{3}$ and porosity $=0.35$.

The data in Figure 3 shows that oil and water removal occur simultaneously during microwave heating, albeit with water removal occurring more readily than oil removal at the same heating time. The amount of oil and water removed increases with heating time, and with applied power. For industrial application $>95 \%$ oil removal is required, and this has been demonstrated in previous studies $[8,10]$. For this study the heating time was restricted to maintain oil and water removal levels $<90 \%$ in order that accurate estimations for steam velocity and mass transfer coefficient can be obtained from the data.

The water and oil removal levels in Figure 3 were subsequently used to establish the rate of steam generation and rate of oil removal, which equate to the slope of the trends within Figure 3. Steam velocity was calculated based on the rate of steam generation (change in water content with time), bed geometry and sample porosity. The mass transfer coefficient for the oil phase was calculated from the oil removal rate (change in measured oil content with time), bed cross-sectional area and vapour pressure of the oil at $100^{\circ} \mathrm{C}$. Table 2 shows the calculated values of steam velocity and mass transfer coefficient for the three power levels and raw data shown in Figure 3.

\begin{tabular}{|c|c|c|c|c|}
\hline Power $(\mathrm{kW})$ & $\begin{array}{c}\text { Steam } \\
\text { generation } \\
\text { rate }(\mathrm{g} / \mathrm{s})\end{array}$ & $\begin{array}{c}\text { Steam Velocity, } \mathrm{u} \\
(\mathrm{m} / \mathrm{s})\end{array}$ & $\begin{array}{c}\text { Oil removal rate } \\
(\mathrm{g} / \mathrm{s})\end{array}$ & $\begin{array}{c}\text { Oil Phase overall } \\
\text { mass transfer } \\
\text { coefficient, } \mathrm{kga}_{\mathrm{ga}}\left(\mathrm{s}^{-1}\right)\end{array}$ \\
\hline 1 & 0.211 & 0.298 & 0.162 & 0.229 \\
\hline 3 & 0.364 & 0.514 & 0.265 & 0.376 \\
\hline 5 & 0.461 & 0.651 & 0.292 & 0.415 \\
\hline
\end{tabular}


Table 2 - Steam velocity and mass transfer coefficients for Shale 1 in system A at power levels of 1$5 \mathrm{~kW}$. Bulk density $=1650 \mathrm{~kg} / \mathrm{m}^{3}$, porosity $=0.35$.

The samples studied were as-received from North Sea drilling operations, hence there was no scope to control the physical properties. In this case, isolating and studying the effect of a single process variable is impossible. Although uncontrolled, the properties were measured prior to each experiment thus enabling a reliable calculation of steam velocity despite the inherent variability of the feedstock. The power levels quoted in Table 2 are forward power values. Reflected power was measured with time during each experiment (typically 5-30\%) and used to calculate the energy input. Energy input is therefore an uncontrolled variable, but one that can be quantified. Table 3 shows the full range of controlled and uncontrolled variables that were measured during this study. In total there were 10 independent variables.

\begin{tabular}{|l|l|l|}
\hline \multirow{2}{*}{ Variable } & \multicolumn{2}{|c|}{ Value } \\
\cline { 2 - 3 } & System A & System B \\
\hline Power (controlled) & $1-5 \mathrm{~kW}$ & $40-100 \mathrm{~kW}$ \\
\hline Heating time (controlled) & $5-45$ seconds & $25-125 \mathrm{~seconds}$ \\
\hline Porosity & $0.29-0.49$ & $0.44-0.48$ \\
\hline Bulk Density & $1400-2100 \mathrm{~kg} / \mathrm{m}^{3}$ & $1500-1600 \mathrm{~kg} / \mathrm{m}^{3}$ \\
\hline Feedstock Oil Content & $8.5-12.0 \% \mathrm{w} / \mathrm{w}$ & $9.0-10.0 \% \mathrm{w} / \mathrm{w}$ \\
\hline Feedstock Water Content & $9.5-14.0 \% \mathrm{w} / \mathrm{w}$ & $7.0-8.0 \% \mathrm{w} / \mathrm{w}$ \\
\hline Operating Frequency (controlled) & $2.45 \mathrm{GHz}$ & $896 \mathrm{MHz}$ \\
\hline Sample mass (controlled) & $70 \mathrm{~g}$ & $6.5 \mathrm{~kg}$ \\
\hline Energy Absorbed & $99-179 \mathrm{kWh} / \mathrm{tonne}$ & $104-210 \mathrm{kWh} / \mathrm{tonne}$ \\
\hline Feedstock Solid Component & Sandstone, Shale 1 & Shale 2 \\
\hline
\end{tabular}

Table 3 - Experimental and process variables studied with System A and System B.

The bed temperature was not used as a measured variable due to the inherent challenges and uncertainties of temperature measurement during microwave heating of heterogeneous materials [19]. Experiments were carried out within the ranges shown in Table 3. In all, 33 separate trends for oil and water removal were obtained for System A and 12 separate trends obtained with System B. The large number of uncontrolled variables mean that a comparison of Systems A \& B based on the controlled variables is not possible. Steam velocity $(\mathrm{u})$ and oil-phase mass transfer coefficient $\left(\mathrm{kg}_{\mathrm{g}}\right)$ were subsequently calculated across the 45 separate sets of experiments containing up to 10 independent variables. The relationship between $\mathrm{u}$ and $\mathrm{kga}$ is shown in Figure 4, for Systems A \& B. 


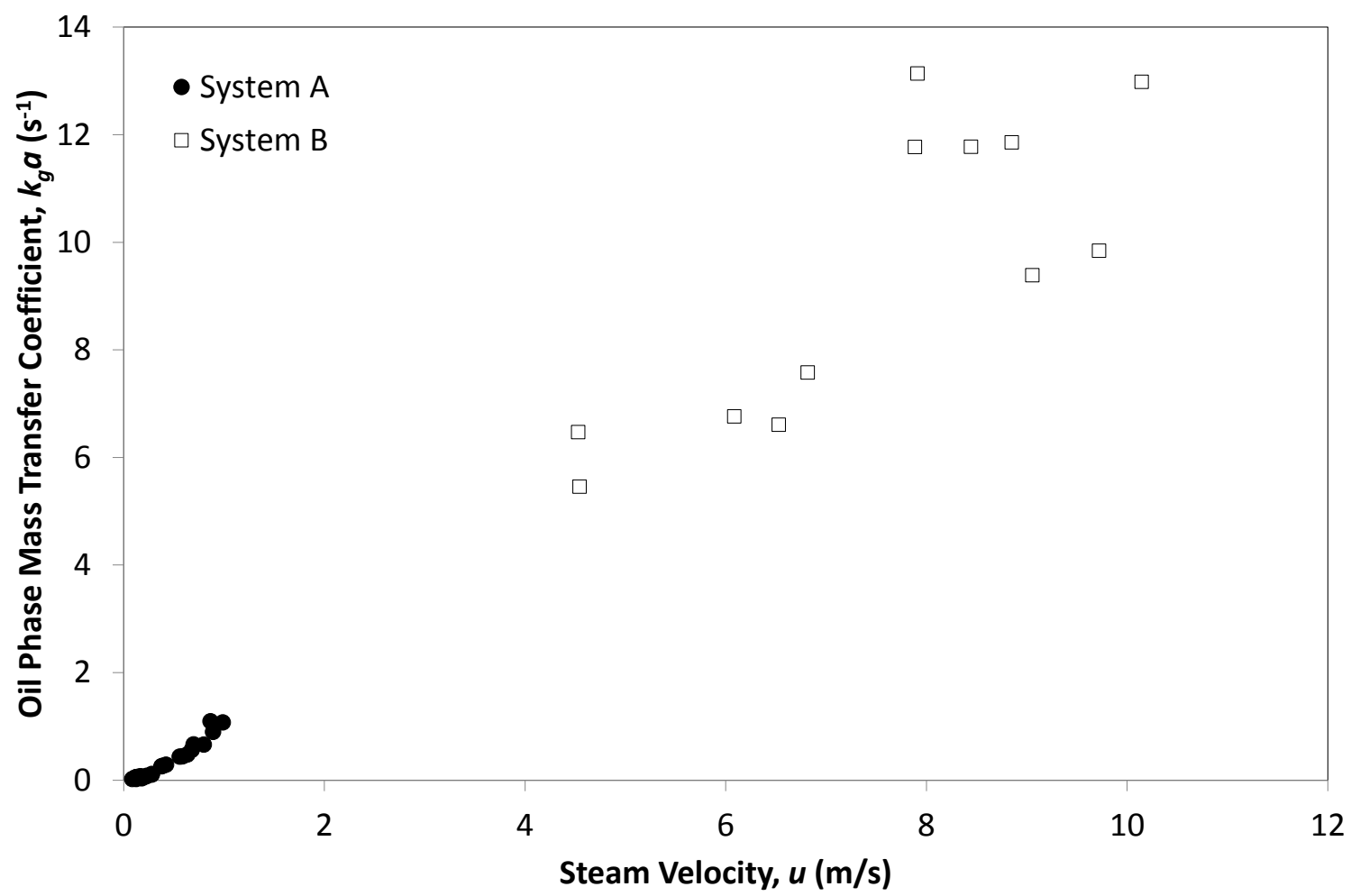

Figure 4 - Relationship between mass transfer coefficient and steam velocity for 10 independent variables across 45 experiments.

A clear correlation exists between $\mathrm{kga}_{\mathrm{ga}}$ and $\mathrm{u}$. Data for System A is within the $0-1 \mathrm{~m} / \mathrm{s}$ velocity range, whereas System B is within the 4-10 m/s range. Despite the use of two different processing systems, each operating at a different frequency, there appears to be good agreement based on the velocity of the produced steam. To assess the contribution of the steam-stripping process to these results the data needs to be presented in a manner that is consistent with the physics of the steam stripping process. Equation 1 can be expanded from the form containing dimensionless groups to one that links $u$ and $k_{g} a:$

$k_{g} a=\frac{D_{a} b}{d_{p}}\left(\frac{u d_{p} \rho}{\mu}\right)^{c} S c^{0.33}$

With no changes in diffusivity, density, viscosity or heat capacity then the only variable terms in Equation 10 are $\mathrm{u}$ and $\mathrm{k}_{\mathrm{g}} \mathrm{a}$. Thus for a steam stripping process to dominate, a plot of $\ln \left(\mathrm{k}_{\mathrm{g}} \mathrm{a}\right)$ versus $\ln (\mathrm{u})$ should yield a straight line with gradient $\mathrm{c}$. The $\ln -\ln$ plot for the experimental data from this study is shown in Figure 5. 


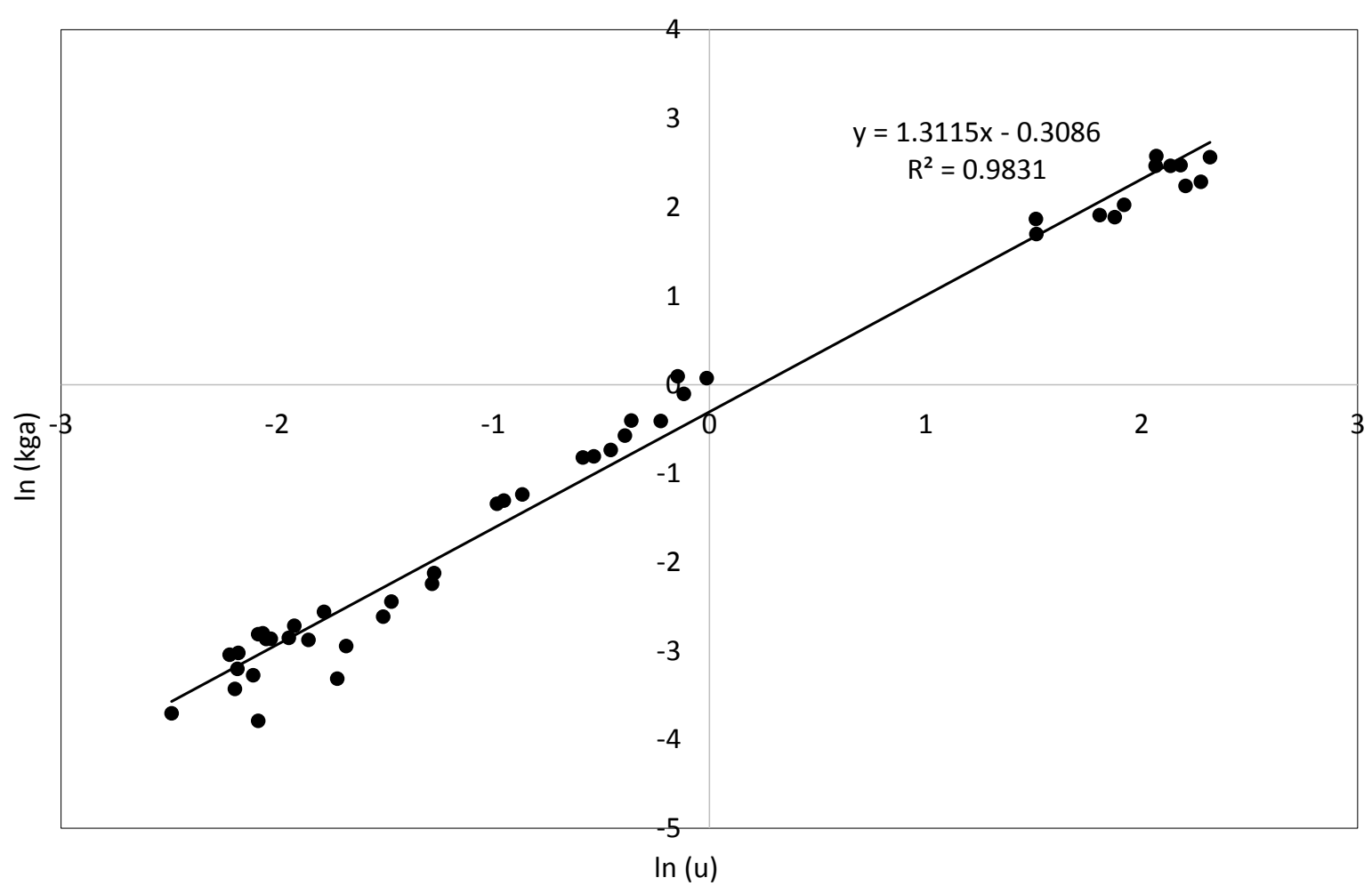

Figure $5-\ln \left(k_{g} a\right)$ plotted against $\ln (u)$ to assess the validity of the steam stripping mechanism and calculation of the exponential factor, $\mathrm{c}$.

Figure 5 shows a positive, linear correlation between $\ln \left(\mathrm{kg}_{\mathrm{g}} \mathrm{a}\right)$ and $\ln (\mathrm{u})$. The ten separate variables that comprise the entire dataset can all be rationalised by a single trend based on the physics of steamstripping. The mass transfer coefficient and velocity relationship obtained from Figure 5 can be given by:

$k_{g} a=0.734 u^{1.31} \quad\left(\mathrm{SEE}=0.26, \mathrm{P}<0.005, \mathrm{R}^{2}=0.9831\right)$

It is clear, therefore, that steam velocity is the major governing factor within the process, which in turn promotes evaporative mass transfer of the hydrocarbon phase. If steam distillation were to be the dominant mechanism then both hydrocarbon and water phases will be removed by boiling, and there would be no dependence on steam velocity. Given the positive correlation obtained in Figure 5 for such a large number of variables it is highly likely that steam stripping is therefore the dominant mechanism that governs hydrocarbon removal from solid materials during microwave processing. Improvements in the efficiency of oil removal (increased $\mathrm{kga}_{\mathrm{g}}$ ) can therefore be achieved by manipulating the velocity of the steam that is generated in-situ from the water that is present within the solid. Increasing steam velocity can be achieved in two ways: 
1. Increasing power density within the solid bed, which increases the rate of steam generation. Power density is related to the square of the electric field intensity, which in turn can be increased by increasing the applied microwave power or by manipulating the geometry of the processing system. Doubling the microwave power doubles the steam velocity, which increases the mass transfer coefficient by a factor of 2.48 .

2. Decreasing the external bed cross-sectional area. This reduces the internal volume and crosssectional area for steam flow, which forces the produced steam to pass through the bed at a higher velocity. If the cross-sectional area is halved then the steam velocity is doubled, increasing $\mathrm{kg}_{\mathrm{g}}$ by a factor of 2.48 .

Previous studies have identified a 'power density' effect, whereby the efficiency of oil removal is improved when higher power levels are applied at an equivalent energy input, although the physical basis for the effect was not fully-explained $[6,8]$. The effect can now be rationalised as a steam stripping mechanism, with higher power densities promoting a higher steam velocity and subsequently increased removal efficiency of the hydrocarbon phase.

\section{Comparison with existing Steam Stripping processes}

The trend obtained in Figure 5 and the subsequent mass transfer correlation shown by Equation 11 can be compared with other known steam-stripping processes within fixed beds. Available data within the open literature data is based on a correlation of Peclet and Sherwood numbers. The Peclet number is a function of the Reynolds number and Schmidt number (Equation 10), with the Schmidt number defined as;

$$
S c=\frac{\mu}{\rho D_{a}}
$$

The Schmidt number is constant in this case since none of the parameters will vary appreciably during microwave treatment. In this case, the Sherwood number varies only with the Peclet number and the mass transfer correlation for the microwave steam-stripping process becomes:

$$
S h_{0} \propto P e^{1.31}
$$

The equivalent correlations from previous studies are shown in Table 2. 


\begin{tabular}{|c|c|c|}
\hline Source & Correlation & $\begin{array}{c}\text { Gas-phase } \\
\text { velocity (m/s) }\end{array}$ \\
\hline Wilkins et al [12] & $S h_{0} \propto P e^{0.62}$ & $0.003-0.015$ \\
\hline $\begin{array}{c}\text { Hongkyu Yoon et al [13] } \\
S h_{0} \propto P e^{0.68}\end{array}$ & $0.005-0.015$ \\
\hline $\begin{array}{c}\text { Van der Ham and } \\
\text { Brouwers [14] }\end{array}$ & $S h_{0} \propto P e^{0.88}$ & $0.045-0.186$ \\
\hline This work & $S h_{0} \propto P e^{1.31}$ & $0.117-9.721$ \\
& & \\
\hline
\end{tabular}

Table 2 - Mass transfer correlations for fixed bed systems.

Previous studies have showed that the exponential factor for the Peclet number is $0.62-0.88$ for evaporative mass transfer within fixed bed systems. The microwave steam stripping process yields an exponential factor of 1.31, far higher than that observed within previous studies, despite appearing to obey the same physical principles (Figure 5). The implication of the higher exponential factor is that the rate of hydrocarbon removal is higher with a microwave heating process compared to a conventional mass transfer process, even at comparable gas velocities. The system reported by Wilkins et al. [12] will yield an $84 \%$ increase in mass transfer coefficient if the steam velocity is doubled, whereas the microwave process will produce a corresponding increase of $148 \%$. The steam velocities in this work span three orders of magnitude, with the lower velocities overlapping with the ranges reported in previous studies. It is also apparent that the upper range of steam velocities obtained during this study are around two orders of magnitude higher than those previously reported. Higher velocities are likely to be obtained with a microwave process due to the volumetric heating and in-situ steam generation, as opposed to the need for a large bulk steam flow with conventional stripping processes. The high velocity and elevated exponential factor are phenomena that appear to be unique characteristics of the microwave heating process, and present significant opportunities for energy and cost savings using this technique. Operating costs can be reduced due to the enhanced efficiency of oil removal, and plant footprint and associated capital costs reduced with the removal of the bulk steam flow and boiler system.

\section{Why does microwave heating enhance mass transfer?}

In conventional mass transfer within a fixed bed the stripping gas is supplied from an external source. In this case it is highly likely that a degree of channelling occurs as the gas passes through the bed [20]. High gas velocities will occur in areas of high voidage, whereas limited gas flow will occur in areas of low voidage and thus the contact between stripping gas and hydrocarbons will be limited within these regions. With microwave heating the stripping gas is steam, which is generated in-situ 
from the water that is present within the solid. Steam is generated throughout the entire bed volume, including areas of low voidage, meaning that the contact between hydrocarbon and stripping gas is more consistent throughout the bed volume and not prone to the channelling effects that occur within conventional gas stripping processes. It is thought therefore that the relationship shown in Figure 5 and the subsequent correlation in Equation 11 could be achieved with a conventional stripping process if channelling could be avoided. The unique characteristic of microwave heating is that the stripping gas is generated in-situ by selective heating of the water, and it is this in-situ steam generation throughout the bed that overcomes the channelling that limits conventional evaporative mass transfer processes. This finding implies that a microwave-based stripping process that promotes in-situ steam generation will be significantly more energy efficient than a conventional stripping process, as energy need only be supplied to raise steam from the water present within the solid rather than to heat the bulk stripping gas.

\section{Conclusions}

It is shown that the dominant mechanism for hydrocarbon removal during microwave heating is evaporative mass transfer due to a steam stripping process, with the steam produced in-situ from selective heating of water within the solid material. Hydrocarbon removal rates were found to be depend solely on the velocity of the steam within the pores of the solid, and a single correlation based on the physics of steam stripping was able to rationalise ten separate experimental variables with $\mathrm{R}^{2}=$ 0.9831 across three orders of magnitude of steam velocity. The microwave-based stripping process was also shown to be more efficient than conventional gas stripping. The mass transfer coefficient is proportional to $\mathrm{u}^{1.31}$ for microwave processing, compared to $\mathrm{u}^{0.62-0.88}$ for conventional gas stripping. The improvement in efficiency is attributed to the ability of microwave heating to overcome channelling, thereby improving contact between the stripping gas and the hydrocarbon phase. This is a unique effect associated with microwave heating, and presents opportunities to improve the efficiency and mass transfer performance in processes where separation of hydrocarbons from solids is required.

\section{Acknowledgements}

EPSRC and NOV WellSite Services are acknowledged for providing the EngD scholarship for Oluwatosin Ogunniran.

\section{Nomenclature}

$a \quad$ Specific Interfacial Area $\left(\mathrm{m}^{2} / \mathrm{m}^{3}\right)$

A Cross-sectional area $\left(\mathrm{m}^{2}\right)$ 
$c \quad$ Gas phase concentration $\left(\mathrm{mol} / \mathrm{m}^{3}\right)$

$d_{p} \quad$ Particle diameter (m)

$D_{a} \quad$ Diffusion coefficient $\left(\mathrm{m}^{2} / \mathrm{s}\right)$

$J \quad$ Hydrocarbon flux $\left(\mathrm{mol} / \mathrm{m}^{2} . \mathrm{s}\right)$

$k_{g} \quad$ Mass transfer coefficient $(\mathrm{m} / \mathrm{s})$

$\dot{N} \quad$ Hydrocarbon molar flowrate $(\mathrm{mol} / \mathrm{s})$

$t \quad$ Time (s)

$u \quad$ Velocity $(\mathrm{m} / \mathrm{s})$

$V \quad$ Bed volume $\left(\mathrm{m}^{3}\right)$

$W \quad$ Water mass $(\mathrm{kg})$

$\varepsilon \quad$ porosity

$\mu \quad$ viscosity (Pa.s)

$\rho \quad$ density $\left(\mathrm{kg} / \mathrm{m}^{3}\right)$

Pe Peclet Number

Re Reynolds Number

Sc Schmidt Number

Sh Sherwood Number

\section{References}

1. Arthur E. Lord Jr, Steam stripping organics from "fluidized" soils (versus confined soils), Journal of Environmental Engineering 124(4) (1998) 390-394.

2. H. Micheal Buettner, William D. Daily, Cleaning contaminated soil using electrical heating and air stripping, Journal of Environmental Engineering 121 (8) (1995) 580-589.

3. Shang Hui, Colin E. Snape, Sam W. Kingman, John P. Robinson, Treatment of Oil contaminated Drill Cuttings by Microwave Heating in a High Power Single-Mode Cavity, Industrial Engineering Chemistry Research 44 (2005) 6837 - 6844

4. Ronald W. Missen, Charles A. Mims, Bradley A. Saville, Introduction to Chemical Reaction Engineering and Kinetics: John Wiley \& Sons Inc, New York., 1999

5. J.P Robinson, S.W Kingman, O. Onobrakpeya, Microwave-assisted stripping of oil contaminated drill cuttings, Journal of Environmental Management 88 (2008) 211-218

6. J.P Robinson, S.W. Kingman, C.E. Snape, S.M. Bradshaw, M.S.A. Bradley, H. Shang, R. Barranco, Scale-up and design of a continuous microwave treatment system for the processing of oil-contaminated drill cuttings, Chemical Engineering Reasearch and Design 88 (2010) 14-154

7. J.P Robinson, S.W Kingman, E.H Lester, C. Yi, Microwave remediation of hydrocarboncontaminated soils - Scale up using batch reactors, Separation and Purification Technology 96 (2012) 12 -19.

8. A.J Buttress, E. Binner, C. Yi, P. Palade, J.P. Robinson, S.W Kingman, Development and evaluation of a continuous microwave processing system for hydrocarbon removal from solids, Chemical Engineering Journal 283 (2016) 215-222 
9. John Robinson, Eleanor Binner, Abdul Saeid, Mohammed Al-Harahsheh, Sam Kingman, Microwave processing of oil sands and contribution of clay minerals, Fuel 135 (2014) 153161.

10. H. Shang, C.E Snape, S.W. Kingman, J.P. Robinson, Microwave treatment of oilcontaminated North Sea drill cuttings in a high power multimode cavity 49 (2006) 84-90.

11. Pingkuan Di, Daniel P.Y Chang, Harry A. Dwyer, Heat and mass transfer during microwave steam treatment of contaminated soils, Journal of Environmental Engineering 126 (2000) 1108-1115.

12. Mark D. Wilkins, Linda M. Abriola, Kurt D. Pennell, An experimental investigation of ratelimited nonaqueous phase liquid volatilization in unsaturated porous media: Steady state mass transfer, Water Resources Research 31 (1995) 2159-2172.

13. Hongkyu Yoon, Joong Hoon Kim, Howard M. Liljestrand, Jeehyeong Khim, Effect of water content on transient nonequilibrium NAPL-gas mass transfer during soil vapour extraction, Journal of contaminant hydrology 54 (2002) 1-18

14. A.G.J van der Ham, H.J.H Brouwers, Modelling and experimental investigation of transient, nonequilibrium mass transfer during steam stripping of a nonaqueous phase liquid in unsaturated porous media, Water resources research 34 (1998) 47-54.

15. J. Comiti, E. Mauret, M. Renaud, Mass transfer in fixed beds: proposition of a generalised correlation based on an energetic criterion, Chemical Engineering Science 55 (2000) 55455554.

16. Thomas K. Sherwood, Robert L. Pigford, Charles R. Wilke, Mass Transfer, McGraw-Hill, U.S.A., 1975.

17. Marijke Huysmans, Alain Dassargues, Review of the use of Peclet numbers to determine the relative importance of advection and diffusion in low permeability environments, Hydrogeology Journal 13 (2004) 895-904.

18. Robert H. Perry, Don W. Green., Perry's Chemical Engineers' Handbook, $8^{\text {th }}$ edition. McGraw-Hill, New York., 2008

19. C. Oliver Kappe and Alexander Stadler. Microwaves in Organic and Medicinal Chemistry. Volume 25 of Methods and Principles in Medicinal Chemistry. Wiley VCH, Weinheim, Germany. 2005.

20. John S Gierke, Neil J Hutzler, David B. Mckenzie, Vapour transport in Unsaturated Soil Columns: Implications for Vapor Extraction, Water Resources Research, 28 (2)(1992) 323335 . 